\title{
NSC-87877 Inhibits Cell Growth by Suppressing ERK Signaling
}

\author{
Mina Song and Sayeon Cho* \\ College of Pharmacy, Chung-Ang University, Seoul 156-756, Korea. *E-mail: sycho@cau.ac.kr \\ Received July 27, 2010, Accepted August 2, 2010
}

Key Words: NSC-87877, Protein tyrosine phosphatase, ERK

Reversible phosphorylation of proteins on tyrosine residues is controlled through the actions of protein tyrosine kinases (PTKs) and protein tyrosine phosphatases (PTPs). ${ }^{1,2}$ Phosphorylation and dephosphorylation of proteins is a key event in signalings that are involved in the control of cell growth, differentiation, adhesion, and death. ${ }^{3-5}$ PTPs can be grouped into four classes: the classical receptor PTPs (RPTPs), the classical non-receptor PTP (nrPTPs), the dual-specificity phosphatases (DUSPs) and the low Mr PTPs. ${ }^{6}$

The mitogen-activated protein kinases (MAPKs) are key signal-transducing enzymes that regulate proliferation, cell survival and death, differentiation, development, immune function, gene expression, and other intracellular events. ${ }^{7}$ In mammals, MAPKs comprise three major groups, which are classified on the basis of sequence similarity, differential activation by agonists, and substrate specificity. These are the c-Jun N-terminal kinases (JNKs), the p38 MAPKs, and the extracellular signalregulated kinases (ERKs). ${ }^{8}$ Of those three MAPK groups, JNK and p38 are involved in cell death whereas ERK induces cell growth. ${ }^{9}$ All of them are activated by hydrogen peroxide $\left(\mathrm{H}_{2} \mathrm{O}_{2}\right)$. Phosphorylation of the threonine and tyrosine residues in an activation loop of the MAPKs results in activation. Thus, dephosphorylation of the MAPKs within the activation loop is important for their control. This could be achieved by serine/ threonine phosphatases, tyrosine phosphatases, or dual-specificity phosphatases.

Numerous studies have presented that modulation of PTP enzymatic activities is critical in regulating disease susceptibility. ${ }^{10}$ Therefore, chemical compounds that regulate the activities of PTP could ultimately lead to the development of novel therapeutics for the treatment of diseases such as cancer, diabetes, obesity, and inflammation.

8-Hydroxy-7-(6-sulfonaphthalen-2-yl)diazenyl-quinoline5-sulfonic acid (NSC-87877) is known as a potent PTP inhibitor with selectivity for Src homology region 2 (SH2) domain-containing phosphatases 2 (SHP-2) and SHP-1. SHP proteins are characterized by containing two SH2 N-terminal domains and a C-terminal protein tyrosine phosphatase domain. SHP-2 is a ubiquitous PTP which plays important biological functions in response to various growth factors, hormones, or cytokines. In particular, SHP-2 is involved in activation of ERK1/2 by epidermal growth factor (EGF). ${ }^{11}$ SHP-2 plays important biological functions in disease pathways such as leukemia, diabetes, neurodegeneration, and cancer. ${ }^{12,13}$

Using in vitro phosphatase assays, we have previously found that DUSP3, DUSP14, DUSP23, DUSP26, ACP1, Cdc25A and Cdc25B are inhibited by NSC-87877. ${ }^{14-19}$ PTPs that are inhibited by NSC-87877 and their specificity for MAPKs were listed in Table 1. Since those NSC-87877 targets are involved in either cell growth or apoptosis, it is of interest to investigate whether cells survive or die when cells are treated with NSC87877. To evaluate an effect of NSC-87877 on cell growth, we analyzed cell growth using the CCK-8 assay. NCI-H460 cells (human lung cancer cells) were preincubated with or without NSC-87877, and then treated with $\mathrm{H}_{2} \mathrm{O}_{2}$ to activate MAPKs. NCI-H460 cell growth was decreased by NSC-87877 (Fig. 1). These data suggest that NSC-87877 might either enhance cell death or inhibit cell growth.

If NSC-87877 enhances cell death signaling, inactivation of PTP activity by NSC-87877 may reduce the phosphorylation of ERKs that are linked to cell survival or enhance the phosphorylation of JNK or p38 that induces cell death. To test our

Table 1

\begin{tabular}{cc}
\hline PTP & MAPK specificity \\
\hline DUSP3 & ERK \\
DUSP14 14, ERK, JNK, p38 \\
DUSP23 2 ERK \\
DUSP26 & p38, ERK \\
ACP1 & No activity \\
Cdc25A, B & No activity \\
SHP-2 & Upregulates ERK signaling \\
\hline
\end{tabular}

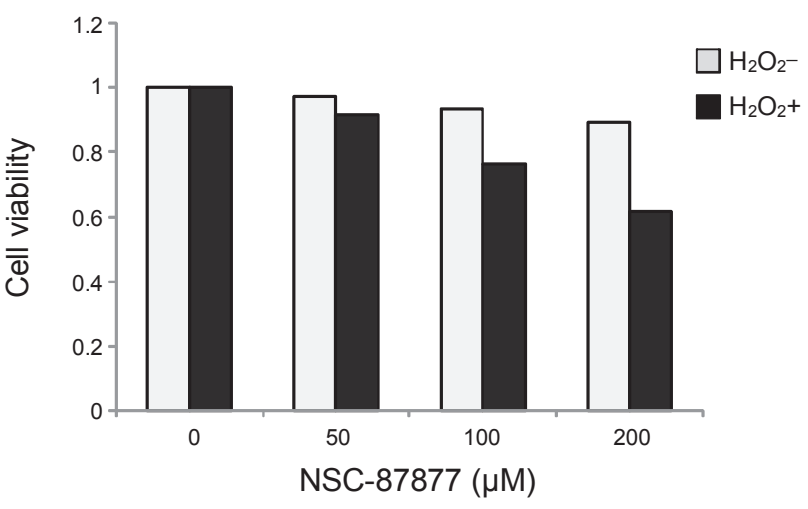

Figure 1. Effects of NSC-87877 on the growth of NCI-H460 cell lines. NCI-H460 cells were cultured for $3 \mathrm{~h}$ at various concentrations of the NSC-87877 and then incubated with or without $0.1 \mathrm{mM} \mathrm{H}_{2} \mathrm{O}_{2}$ for $16 \mathrm{~h}$. Cell growth was assessed by CCK-8 cell proliferation assay method and is expressed as a percentage of control. 


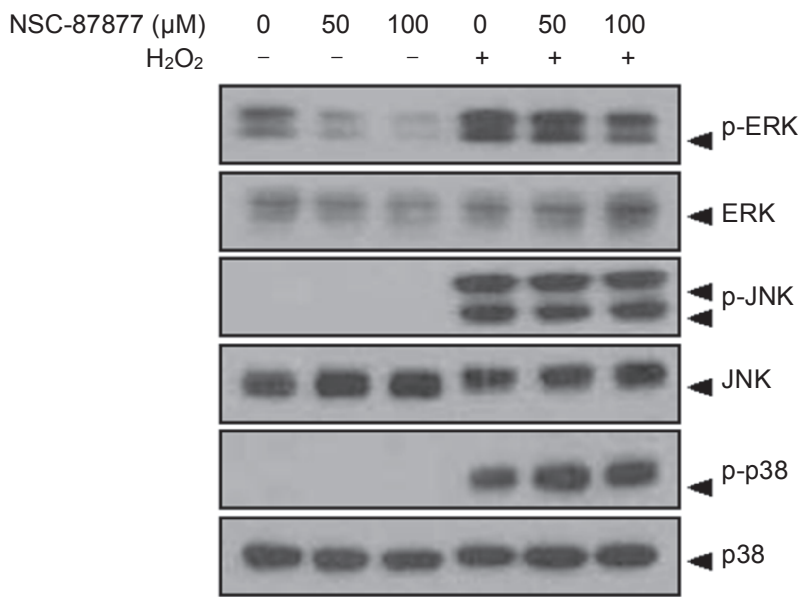

Figure 2. NSC-87877 inhibits ERK1/2 activation. NCI-H460 cells were pretreated with various concentrations of NSC-87877 for $3 \mathrm{~h}$ and then stimulated with $0.1 \mathrm{mM} \mathrm{H}_{2} \mathrm{O}_{2}$. MAPKs activation was analyzed by Western blotting of cell lysates with antibodies to phosphorylated MAPKs.

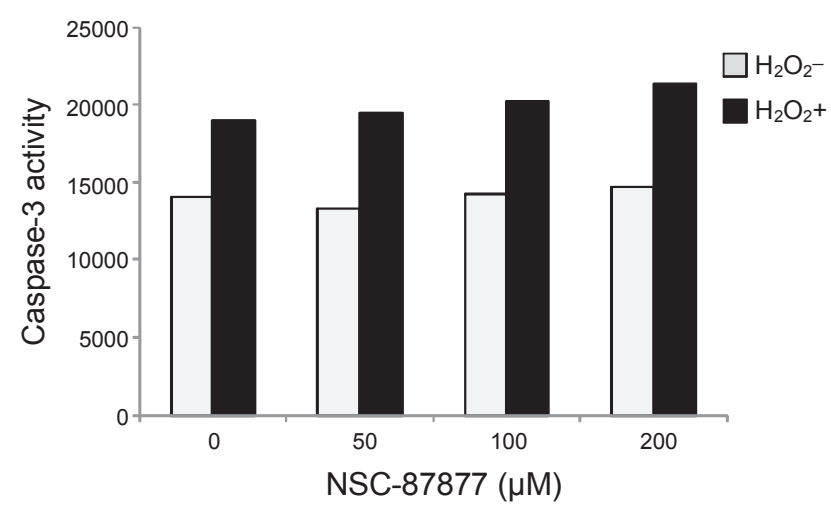

Figure 3. Caspase-3 activity in NCI-H460 cells in the presence of NSC-87877. NCI-H460 cells were pretreated with various NSC87877 concentrations as indicated and then incubated with or without $0.1 \mathrm{mM} \mathrm{H}_{2} \mathrm{O}_{2}$ for $16 \mathrm{~h}$. Caspase- 3 activity was determined using the Caspase-3 assay kit as described under "Experimental section".

hypothesis, we examined effects of NSC-87877 on endogenous MAPK phosphorylation. As shown in Fig. 2, NSC-87877 inhibits $\mathrm{H}_{2} \mathrm{O}_{2}$-induced ERK1/2 phosphorylation. In contrast, phosphorylation levels of JNK and p38 by NSC-87877 were not changed. These results indicate that ERK phosphorylation is involved in the growth inhibition mediated by NSC- 87877 .

We performed caspase- 3 assays to see if NSC-87877 induces cells to undergo apoptosis. NCI-H460 cells were pre-treated with or without the NSC-87877 prior to $\mathrm{H}_{2} \mathrm{O}_{2}$ treatment. Caspase- 3 assays with cell lysates showed that NSC-87877 treatment did not induce apoptosis (Fig. 3). Thus, our results suggest that treatment with NSC-87877 leads to cell growth inhibition through dephosphorylation of ERKs rather than apoptosis.

In the present study, we found that NSC-87877 was effective in ERK dephosphorylation and inhibited cell proliferation. Even though NSC-87877 has the PTP inhibitory activity against several human PTPs in vitro, NSC-87877 seemed to be involved with inhibition of SHP-2 in cell-based assays since SHP-2 activity is necessary for ERK activation. Since SHP-2 is an important molecule for controlling growth factor receptor signaling and a potential target for development of novel therapies for SHP2-associated disease, this study provides potential pharmacological role of NSC-87877.

\section{Experimental Section}

Cell culture and transfection. The NSCLC cell lines NCI$\mathrm{H} 460\left(\mathrm{p} 53^{+/+}\right)$were maintained at $37^{\circ} \mathrm{C}$ in Roswell Park Memorial Institute (RPMI) medium 1640 (Invitrogen, Carlsbad California) supplemented with $10 \%$ fetal bovine serum (FBS, Invitrogen) and penicillin/streptomycin in the presence of 5\% $\mathrm{CO}_{2}$.

Reagents and antibodies. Polyclonal anti-JNK, anti-phospho-JNK (Thr-183/Tyr-185), anti-p38, anti-phospho-p38 (Thr180/Tyr-182), anti-ERK1/2, and anti-phospho-ERK1/2 (Thr202/Tyr-204) antibodies were from Cell Signaling Technology (Danvers, MA). The NSC-87877 was from Calbiochem (San Diego, CA).

Westem blotting analysis. Western blotting was carried out as previously described. ${ }^{15}$

Cell viability assay. Cell viability was determined using a CCK-8 cell viability assay kit (DOJINDO Laboratories, Japan). NCI-H460 cells $\left(5 \times 10^{3}\right.$ cells/well) were pretreated with various NSC-87877 concentrations as indicated and then incubated with or without $0.1 \mathrm{mM} \mathrm{H}_{2} \mathrm{O}_{2}$ for $16 \mathrm{~h}$ in a 96 -well plate. $10 \mu \mathrm{L}$ of cell viability assay kit solution was added to each well of the plate. After incubation for $1 \mathrm{~h}$ at $37^{\circ} \mathrm{C}$ in the dark, absorbances were measured at $450 \mathrm{~nm}$ using a multiwell plate reader.

Measurement of caspase-3 activity. Caspase-3 assay kit (Peptron, Korea) was used for the detection of caspase-3 activity following the manufacturer's protocol. The subconfluent cells in $100 \mathrm{~mm}$ dishes were pretreated with various NSC-87877 concentrations as indicated and then incubated with or without $0.1 \mathrm{mM} \mathrm{H}_{2} \mathrm{O}_{2}$ for $16 \mathrm{~h}$.

Acknowledgments. This work was supported by a grant of the Korea Health 21 R\&D Project, Ministry of Health \& Welfare, Republic of Korea (A010385-1033-0000400) and by the Korea Science and Engineering Foundation (KOSEF) grant funded by the Korea government (MEST) (No. 2009-0072827).

\section{References}

1. Ostman, A.; Bohmer, F. D. Trends Cell Biol. 2001, 11, 258.

2. Schlessinger, J. Cell 2000, 103, 211.

3. Hunter, T. Curr. Opin. Cell. Biol. 1989, 1, 1168.

4. Ullrich, A.; Schlessinger, J. Cell 1990, 61, 203.

5. Yarden, Y.; Ullrich, A. Annu. Rev. Biochem. 1988, 57, 443.

6. Alonso, A.; Sasin, J.; Bottini, N.; Friedberg, I.; Osterman, A.; Godzik, A.; Hunter, T.; Dixon, J.; Mustelin, T. Cell 2004, 117, 699.

7. Dickinson, R. J.; Keyse, S. M. J. Cell. Sci. 2006, 119, 4607.

8. Cohen, P. Trends Cell Biol. 1997, 7, 353.

9. Xia, Z.; Dickens, M.; Raingeaud, J.; Davis, R. J.; Greenberg, M. E. Science 1995, 270, 1326

10. Marti, F.; Krause, A.; Post, N. H.; Lyddane, C.; Dupont, B.; Sadelain, M.; King, P. D. J. Immunol. 2001, 166, 197.

11. Deb, T. B.; Wong, L.; Salomon, D. S.; Zhou, G.; Dixon, J. E.; Gut- 
kind, J. S.; Thompson, S. A.; Johnson, G. R. J. Biol. Chem. 1998, 273, 16643.

12. Bentires-Alj, M.; Paez, J. G.; David, F. S.; Keilhack, H.; Halmos, B.; Naoki, K.; Maris, J. M.; Richardson, A.; Bardelli, A.; Sugarbaker, D. J.; Richards, W. G.; Du, J.; Girard, L.; Minna, J. D.; Loh, M. L.; Fisher, D. E.; Velculescu, V. E.; Vogelstein, B.; Meyerson, M.; Sellers, W. R.; Neel, B. G. Cancer Res. 2004, 64, 8816.

13. Chong, Z. Z.; Maiese, K. Histol. Histopathol. 2007, 22, 1251.

14. Park, S. J.; Song, M.; Cho, S. Bull. Korean Chem. Soc. 2009, 30,
3098.

15. Song, M.; Cho, S. Bull. Korean Chem. Soc. 2009, 30, 1190.

16. Song, M.; Park, J. E.; Park, S. G.; Lee, H.; Choi, H. K.; Park, B. C.; Ryu, S. E.; Kim, J. H.; Cho, S. Biochem. Biophys. Res. Commun. 2009, 381, 491.

17. Song, M.; Cho, S. Bull. Korean Chem. Soc. 2009, 30, 1858.

18. Song, M.; Cho, S. Bull. Korean Chem. Soc. 2009, 30, 924.

19. Song, M.; Park, S. J.; Cho, S. Bull. Korean Chem. Soc. 2008, 30, 236. 